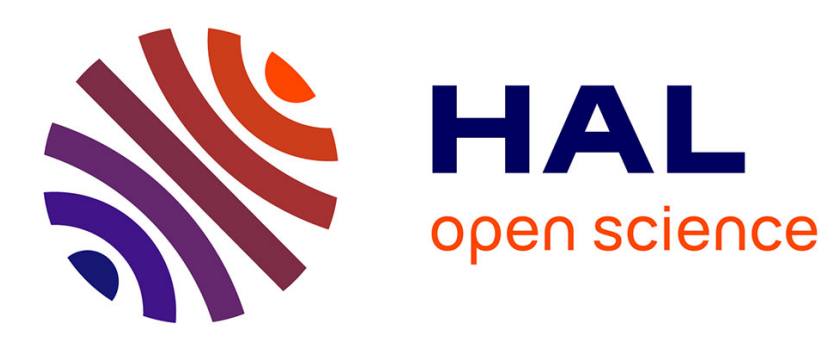

\title{
NMR measurement of self-diffusion coefficients by slice selection
}

Sebastien Leclerc, Gregory Trausch, Jean-Marie Escanye, Daniel Canet

\section{To cite this version:}

Sebastien Leclerc, Gregory Trausch, Jean-Marie Escanye, Daniel Canet. NMR measurement of selfdiffusion coefficients by slice selection. Journal of Chemical Physics, 2004, 121 (1), pp.405-408. 10.1063/1.1756572 . hal-00125368

\section{HAL Id: hal-00125368 \\ https://hal.science/hal-00125368}

Submitted on 29 May 2017

HAL is a multi-disciplinary open access archive for the deposit and dissemination of scientific research documents, whether they are published or not. The documents may come from teaching and research institutions in France or abroad, or from public or private research centers.
L'archive ouverte pluridisciplinaire HAL, est destinée au dépôt et à la diffusion de documents scientifiques de niveau recherche, publiés ou non, émanant des établissements d'enseignement et de recherche français ou étrangers, des laboratoires publics ou privés. 


\section{NMR measurement of self-diffusion coefficients by slice selection}

S. Leclerc, G. Trausch, J.-M. Escanyé, and D. Canet

Citation: The Journal of Chemical Physics 121, 405 (2004); doi: 10.1063/1.1756572

View online: http://dx.doi.org/10.1063/1.1756572

View Table of Contents: http://aip.scitation.org/toc/jcp/121/1

Published by the American Institute of Physics

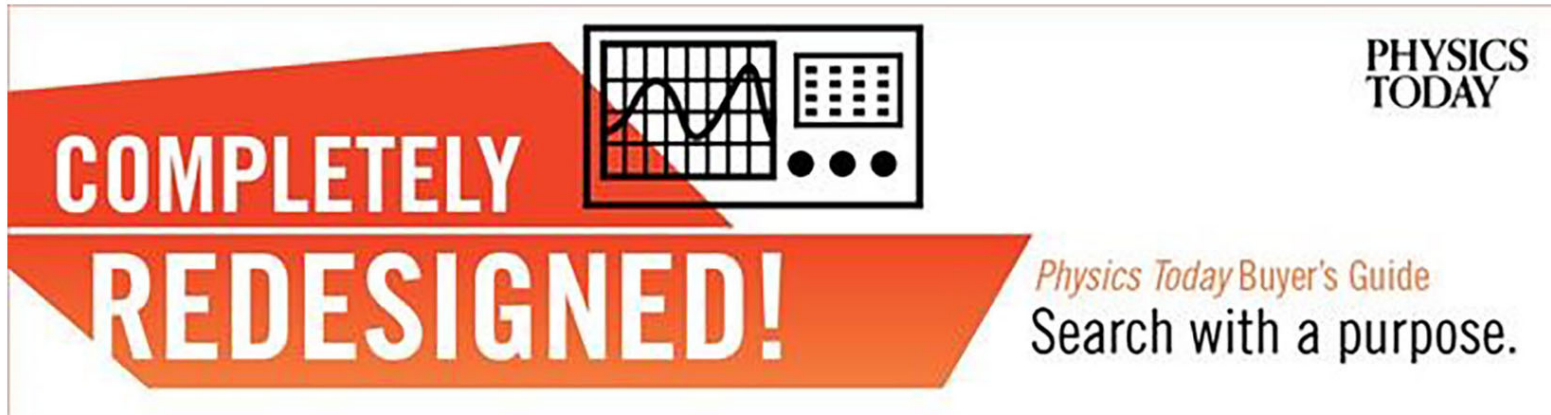




\title{
NMR measurement of self-diffusion coefficients by slice selection
}

\author{
S. Leclerc, G. Trausch, J.-M. Escanyé, and D. Canet ${ }^{\mathrm{a})}$ \\ Méthodologie RMN, Université Henri Poincaré, Nancy I, B.P. 239 54506-Vandoeuvre-les-Nancy \\ (cedex), France
}

(Received 2 April 2004; accepted 6 April 2004)

\begin{abstract}
Most of the time, so-called inversion-recovery experiments concern longitudinal nuclear magnetization of the whole sample, the region of interest being limited by the transmittingreceiving coil. Here we address the question of what occurs if the region of interest is purposely limited to a thin slice selected by means of procedures employing magnetic field gradients. Gradients of both magnetic fields $\left(B_{0}\right.$, the static magnetic field, and $B_{1}$, the radio-frequency magnetic field) can be used. In this study we resorted essentially to $B_{1}$ gradients and novel procedures, based on the natural inhomogeneity of the $B_{1}$ field delivered by a saddle coil, are described. It is obvious that molecules leaving and entering the slice during the evolution (recovery) period should influence the magnetization recovery. Molecular self-diffusion is responsible for such effects, experimentally visible and accounted for by an appropriate theory which has been approximated for by permitting an easy physical assessment. This approach should lead to alternative methods for measuring self-diffusion coefficients. (c) 2004 American Institute of Physics. [DOI: 10.1063/1.1756572]
\end{abstract}

\section{INTRODUCTION}

Slice selection is usually related to two-dimensional NMR images which are generally preferred to threedimensional images in order to save measurement time. Only one or several slices perpendicular to the third dimension are examined. Slice selection ${ }^{1,2}$ is usually achieved by radiofrequency (rf) selective pulses under the application of a static field gradient ( $B_{0}$ gradient). Alternatively, one can resort to radio-frequency field gradients ${ }^{3}$ ( $B_{1}$ gradients) or even to the natural inhomogeneity of a saddle-shaped coil. ${ }^{4}$ We address here the possibility of taking advantage of these experimental procedures in view of applications in other domains than imaging.

The idea underlying the present work concerns selfdiffusion coefficients, which, as far as NMR is concerned, are usually measured by the Stejskal-Tanner method ${ }^{5}$ (also called PGSE for pulsed gradient spin echo) or, alternatively, by a sequence involving simply two $B_{1}$ gradient pulses ${ }^{6}$ which proved to be formally equivalent to the stimulated echo method. ${ }^{7}$ All these experiments employ two gradient pulses separated by a time interval $\Delta$ : spatial labeling (and eventually magnetization defocusing) is achieved by the first pulse whereas the second one produces reverse spatial labeling and therefore refocusing, which is only partial due to translational motions of molecules which bear the considered nuclear spins. In the case of unrestricted self-diffusion, the signal attenuation is given by the well-known formula

$$
S(\Delta)=S(0) K \exp \left(-\gamma^{2} G^{2} \delta^{2} D \Delta\right),
$$

where $\gamma$ is the gyromagnetic ratio of the relevant nucleus, $G$ the gradient amplitude, $\delta$ the duration of the gradient pulse

\footnotetext{
${ }^{a)}$ Author to whom correspondence should be addressed. Electronic mail: Daniel.Canet@rmn.uhp-nancy.fr
}

(supposed to be much smaller than $\Delta$ ), and $D$ the selfdiffusion coefficient. $K$ is a factor (smaller than 1) representing signal attenuation by relaxation phenomena.

The idea of using slice selection is reminiscent of the tracer method. Rather than inserting radioactive labels into a given slice and thereafter following their departure (from this slice), we propose a space encoding based on selective inversion of the nuclear magnetization within the relevant slice followed by selective observation of its recovery towards thermal equilibrium. In the absence of self-diffusion, this is the classical inversion-recovery experiment. If, however, the slice is sufficiently thin, we can expect effects arising from translational motions which result in noninverted spins entering the considered slice and, at the same time, inverted spins leaving it. Altogether, this should increase recovery towards thermal equilibrium. It can be noticed that the proposed method is quasi-independent of the transverse relaxation time $T_{2}$. As for the stimulated echo method, this can be advantageous whenever $T_{1} \gg T_{2}$ (as this is the case for numerous systems). As a matter of fact, $T_{2}$ plays a role only during the slice selection procedure. This must be related to the stimulated echo experiment ${ }^{7}$ for which $T_{2}$ plays a role only during the application of the gradient.

\section{THEORY}

The theory underlying the effects described above can be relatively simply worked out in the case of unrestricted diffusion for which the probability density $P(x / \Delta)$ that the molecule bearing the considered spins has undergone a displacement of length $x$ during the interval $\Delta$ can be written as

$$
P(x / \Delta)=\frac{1}{\sqrt{4 \pi D \Delta}} \exp \left(-\frac{x^{2}}{4 D \Delta}\right),
$$

with 


$$
\int_{-\infty}^{+\infty} P(x, \Delta) d x=1
$$

and

$$
P(x / 0)=\delta(x),
$$

where $\delta(x)$ is a Dirac distribution.

Let us now consider a slice of thickness $2 a$ centered on $x=0$, and let us define the following integral which represents the relative number (proportion) of spins which remain in the considered slice during the time interval $\Delta$ :

$$
\begin{aligned}
I(2 a, D, \Delta)= & \frac{1}{2 a \sqrt{4 \pi D \Delta}} \\
& \times \int_{-a}^{a} \int_{-a}^{+a} \exp \left[-\frac{\left(x^{\prime}-x\right)^{2}}{4 D \Delta}\right] d x d x^{\prime} .
\end{aligned}
$$

Equation (5) represents indeed a proportion due to the factor $2 a$ in the denominator, the latter corresponding to the number of spins in the considered slice at time zero:

$$
\begin{aligned}
& \int_{-a}^{+a} \int_{-a}^{+a} P\left(\left(x^{\prime}-x\right) / 0\right) d x d x^{\prime} \\
& \quad=\int_{-a}^{+a} \int_{-a}^{+a} \delta\left(x^{\prime}-x\right) d x d x^{\prime}=2 a .
\end{aligned}
$$

We have now to introduce the effect of longitudinal relaxation. $k$ will denote the inversion rate $(-1 \leqslant k \leqslant 1$, with $k$ $=1$ for a complete inversion) and $T_{1}$ the longitudinal relaxation time. We have also to account for the spins which entered the slice during the evolution interval. As the density does not change, their relative number is simply 1 $-I(2 a, D, \Delta)$. Altogether, denoting by $S(\Delta)$ and $S(0)$ the measured signals, we obtain

$$
\frac{S(\Delta)}{S(0)}=\frac{(1+k) \exp \left(-\Delta / T_{1}\right) I(2 a, D, \Delta)-1}{k} .
$$

It can be noticed that when $a$ tends toward infinity (experiment performed on the whole sample), $I(2 a, D, \Delta)$ becomes equal to 1 so that one retrieves the well-known expression corresponding to a standard inversion recovery experiment. Of course, it can be expected that the thinner the slice, the more important will be diffusion effects. This is confirmed by numerical evaluations of Eq. (5) or, even better, by an approximation of this integral which is derived below and which proved to be indistinguishable from simulations.

Let $A$ be defined as

$$
A=\frac{a}{\sqrt{2 D \Delta}},
$$

and let $y=x / \sqrt{2 D \Delta}, y^{\prime}=x^{\prime} / \sqrt{2 D \Delta}$; one has, with $\nu=y$ $-y^{\prime}$,

$$
I(2 a, D, \Delta)=\frac{1}{2 A} \int_{-A}^{A}\left(\frac{1}{\sqrt{2 \pi}} \int_{-A-y^{\prime}}^{A-y^{\prime}} e^{-\nu^{2} / 2} d \nu\right) d y^{\prime} .
$$

As the quantity between parentheses is representative of a standard normal distribution, it can be noticed that it tends toward unity if the integral limits tend toward infinity-that is, if $a$ tends toward infinity. Moreover, because $A$ is large [with the usual values of the parameters involved in Eq. (7)], the integral $I$ can be approximated as

$$
\begin{aligned}
I(2 a, D, \Delta) & \approx 1-\frac{1}{A} \int_{0}^{A}\left(\frac{1}{\sqrt{2 \pi}} \int_{A-y^{\prime}}^{\infty} e^{-\nu^{2} / 2} d \nu\right) d y^{\prime} \\
& \approx 1-\frac{1}{A} \int_{A-2.5}^{A}\left(\frac{1}{\sqrt{2 \pi}} \int_{A-y^{\prime}}^{\infty} e^{-\nu^{2} / 2} d \nu\right) d y^{\prime} .
\end{aligned}
$$

The value 2.5 (actually 2.56) has been chosen by reference to the standard normal distribution, the integral of which is equal to 0.99 for the interval $[-2.56,2.56]$. The right-handside approximation in Eq. (9) arises from the fact that (i) $A$ is in most situations greater than 2.5 and (ii) the integral between parentheses has a non-negligible value only for $y^{\prime}$ in the interval $[A-2.5, A]$. Finally, using the numerical values of the error function (the integral between parentheses is related to the complementary error function), we arrive at

$$
I(2 a, D, \Delta) \approx 1-0.598 \frac{\sqrt{D}}{a} \sqrt{\Delta} .
$$

Thus Eq. (10) makes it clear that the experiment is sensitive to the ratio $\sqrt{D} / a$, which is generally lower than unity.

Using Eq. (10), Eq. (6) can be made linear with respect to $\sqrt{D}$, provided that we define $S(0)$ as $-k S_{0}$ where $S_{0}$ is the (equilibrium) signal obtained for $\Delta \rightarrow \infty$ (a quantity easily measured in practice by running an experiment with a value of $\Delta$ sufficiently large with respect to the longitudinal relaxation time $T_{1}$ ). We obtain

$$
\frac{1-S(\Delta) / S_{0}}{e^{-\Delta / T_{1}}}=(1+k)-(1+k) \frac{0.598 \sqrt{\Delta}}{a} \sqrt{D} .
$$

In view of the exploitation of experimental data, Eq. (11) can be written as

$$
Y=A X+B,
$$

with

$$
\begin{aligned}
& Y=\frac{1-S(\Delta) / S_{0}}{e^{-\Delta / T_{1}}}, \quad X=\sqrt{\Delta}, \\
& A=-\frac{0.598(1+k) \sqrt{D}}{a}, \quad B=(1+k) .
\end{aligned}
$$

The slope $A$ and intercept $B$ can be obtained by a simple linear regression ( $T_{1}$ is assumed to be determined from an independent measurement), so that the self-diffusion coefficient is determined from the following expression (the slice thickness being evaluated by the simulations described previously $^{8}$ or, alternatively, by measurements carried out with a compound of known self-diffusion coefficient):

$$
D=\frac{a^{2} A^{2}}{(0.598 B)^{2}} \text {. }
$$




\section{SLICE SELECTION BY AN INHOMOGENEOUS RADIO-FREQUENCY FIELD}

Although experiments reported here could have been performed with static field $\left(B_{0}\right)$ gradients, we have been using, for the sake of simplicity, the inhomogeneity of the radio-frequency field $\left(B_{1}\right)$. The radio-frequency field is more or less inhomogeneous, depending on the NMR probe design; this feature is unwanted for most experiments, explaining why manufacturers make much effort to reduce it to a minimum. By contrast, our objective is here to take advantage of it and to show that the present experiment can be performed with a basic NMR spectrometer devoid of a gradient system. It must, however, be mentioned that, with a standard coil, a relatively modest slice thickness (of the order of $2 \mathrm{~mm}$ ) can only be achieved; improvements are possible with a nonstandard coil geometry (see below).

The methodology to select a defined slice is based on DANTE-like sequences ${ }^{4}$ of the form

$$
\left[(\alpha)_{x}(2 \pi)_{y}\right]_{n} .
$$

Here $x$ and $y$ refer to the radio-frequency phase (in other words, to axes of the so-called rotating frame) whereas the flip angles $\alpha$ and $2 \pi$ are those at the center of the coil of the NMR probe, acknowledging the fact that the radio-frequency field is maximum at this position and presents a strong variation (which can usually be modeled as a Gaussian function) along the coil axis. $n$ is chosen such as $n \alpha=\pi / 2$ or $\pi$ so that the effect of sequence (14) is to drive the magnetization at the coil center toward the desired direction, either $y$ or $-z$, whereas magnetization belonging to other locations is (in principle) scattered in the $(x, z)$ plane of the rotating plane. This is because the flip angles $\alpha$ are cumulative only for magnetization at the coil center (where $B_{1}$ is maximum) as it undergoes exactly a complete revolution due to $(2 \pi)_{y}$ contrary to magnetization of other regions. In fact, the latter is somewhere (preferably near the $z$ axis as this is always the case for DANTE sequences) with possibly (as shown by simulations) a component along $y$, which can pose severe problems of corruption. It would be better to take unselected magnetization toward a well-defined direction-namely, the equilibrium direction $z$. This is accomplished by a simple trick represented by the following sequence which substitutes to Eq. (14):

$$
\left[(\alpha)_{x}(2 \pi)_{y}\right]_{n / 2}\left[(\alpha)_{x}(2 \pi)_{-y}\right]_{n / 2} .
$$

The above pulse sequence has the virtue of refocusing unwanted magnetization components along the $z$ axis, thus avoiding any corruption along the detection axis. Concerning inversion in the selected slice, the efficiency of sequence (15) is illustrated by the simulated profile shown in Fig. 1, which is particularly clean with side lobes reduced to a very small amount. The selective read pulse (for signal measurement) is constructed along the same lines. It turns out that, generally, for a given slice thickness more cycles are needed for selective detection than for selective inversion. Altogether, this indicates that the theory developed in the previous section can be properly checked with methodology employing $B_{1}$ inhomogeneity as demonstrated below.

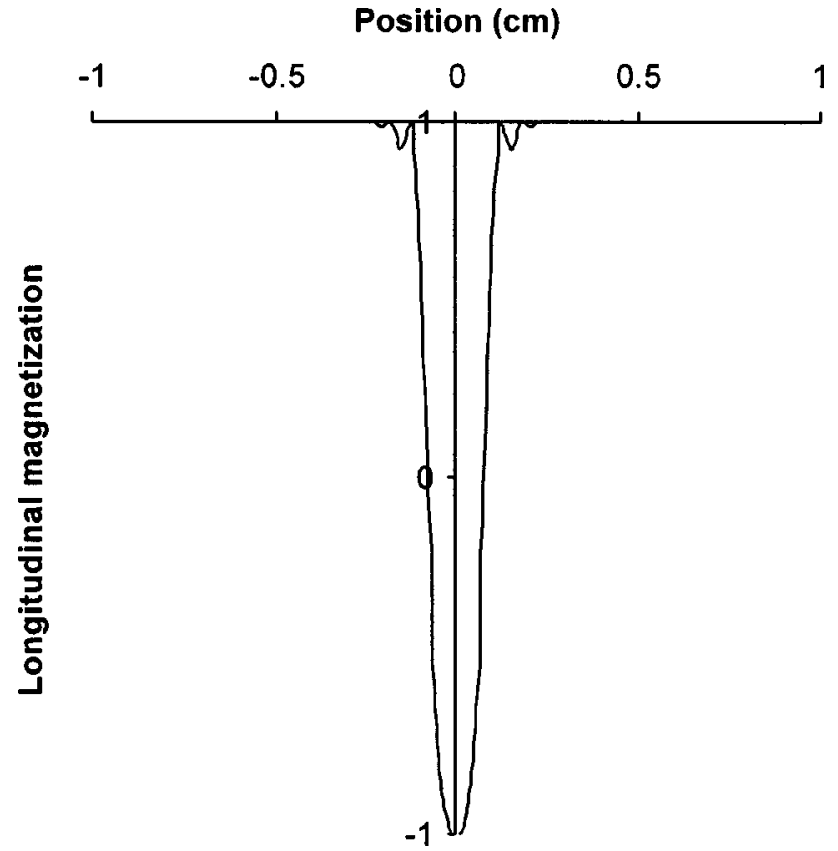

FIG. 1. Profile of the longitudinal magnetization (simulation performed according to methods developed in Ref. 4) corresponding to the application of sequence (15) with $n \alpha=\pi$. For this simulation, the $B_{1}$ field profile was assimilated to a Gaussian function (in view of experimental determination of the actual amplitude of $B_{1}$ along the considered axis) with a standard deviation of $1.5 \mathrm{~mm}$ and $n$ was set at 64 .

\section{EXPERIMENTAL VERIFICATIONS}

All proton NMR measurements have been performed at $200 \mathrm{MHz}$ with a homemade spectrometer. The coil of the NMR probe is of the saddle-shaped type with a geometry designed for enhancing $B_{1}$ inhomogeneity (diameter $8 \mathrm{~mm}$; height $2 \mathrm{~mm}$ ). Here $(2 \pi)$ pulses were applied with the usual amplifier output power (duration $45 \mu \mathrm{s}$ ) whereas for $(\alpha)$ pulses we employed a reduced power so that the pulse duration was never below $2 \mu$ s. Simulations permitted us to adjust the experimental parameters of sequence (15) so that the same slice is involved either for the selective inverting procedure or at the signal measurement stage (slice selective

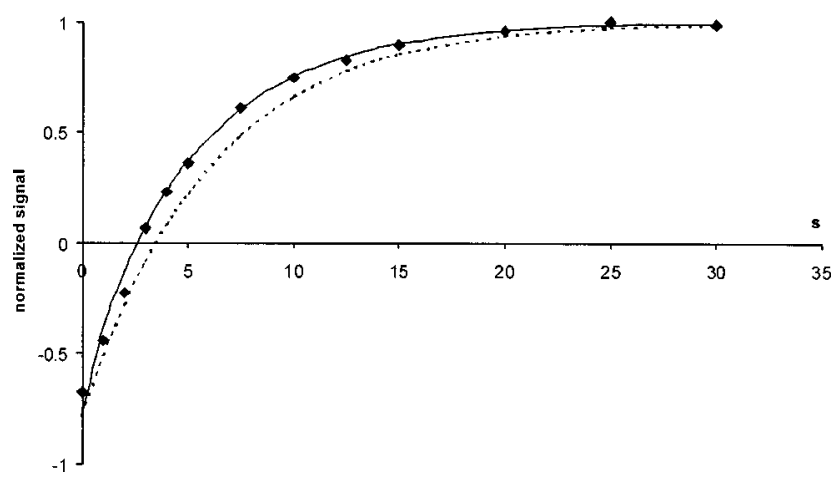

FIG. 2. Proton inversion-recovery curves for a water sample at ambient temperature. Dashed curve: standard experiment without slice selection. Diamonds: experimental data points for an inversion-recovery experiment with the same slice selection for both inversion and signal measurement. The solid curve corresponds to a fit according to Eq. (6). Estimated slice thickness: $600 \mu \mathrm{m}$. For slice selective inversion, $n$ was set at 30 and at 40 for slice selective observation. 


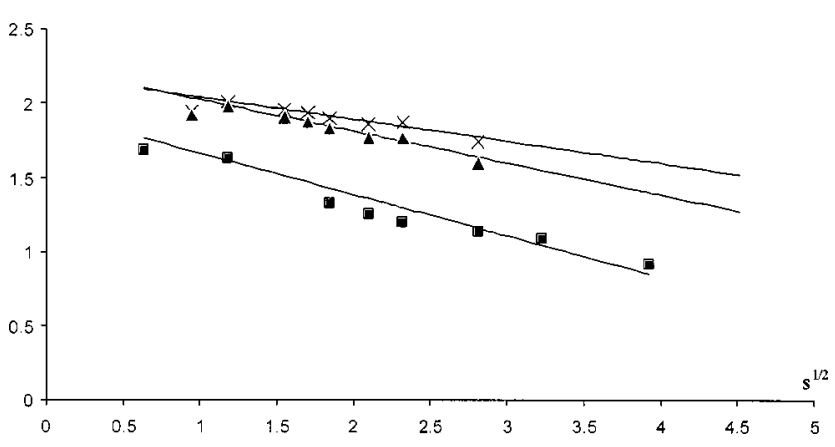

FIG. 3. Proton experimental data presented according to Eqs. (11) and (12): $\left[1-S(\Delta) / S_{0}\right] / e^{-\Delta / T_{1}}$ as a function of $\Delta$. Crosses: ethanol. Triangles: water. Squares: acetone. Same parameters for slice selection as in Fig. 2.

read pulse). Unwanted transverse magnetization which may corrupt experimental results at short $\Delta$ values have to be eliminated. This can be achieved by a crusher $B_{0}$ gradient pulse (in the present experimental setup through an activation of the shim coils).

In order to visualize clearly the effect of molecular diffusion, the inversion recovery curves for water proton magnetization, with and without slice selection, are displayed in Fig. 2. The ability of the method for measuring self-diffusion coefficients was checked with three samples investigated at ambient temperature: namely, acetone, water, and ethanol. Results, presented according to Eqs. (11) and (12) are shown in Fig. 3 along with the best linear fit. Diffusion coefficients can then be derived by applying Eq. (13). We find, in agreement with literature values, $1.2 \times 10^{-9}, 2.3 \times 10^{-9}$, and 5.1 $\times 10^{-9} \mathrm{~m}^{2} \mathrm{~s}^{-1}$ for ethanol, water, and acetone, respectively. It is almost obvious that, at the present time, these results are probably less accurate than those obtained with, for example, the PGSE method, although it is difficult to provide a valid comparison with existing methods because we did not rely on more elaborated procedures (base on $B_{0}$ gradients) which would provide much thinner slices and then improve the measurement quality.

\section{CONCLUSION}

This is a report on a quite novel methodology aimed at measuring self-diffusion coefficients by NMR. As far as the slice thickness is concerned and as already mentioned, improvements can be envisioned for instance by employing $B_{0}$ gradients (preliminary experiments have already proved to be quite successful). Initial perturbations different from the one used here (inversion of the selected slice magnetization) could also be tried.

The method, still in its infancy, might be valuable if strong gradients are required within the frame of classical approaches (e.g., the Stesjkal-Tanner sequence with $B_{0}$ gradients $^{1,2}$ or its equivalent with $B_{1}$ gradients). ${ }^{3}$ It can indeed be anticipated that it is easier to create a nonequilibrium state within a thin slice than applying strong gradient pulses with all the experimental and instrumental artifacts that inevitably affect such experiments.

${ }^{1}$ R. Kimmich, NMR: Tomography, Diffusometry, Relaxometry (Springer, Berlin, 1997).

${ }^{2}$ B. Blümich, NMR Imaging of Materials (Clarendon Press, Oxford, 2000).

${ }^{3}$ D. Canet, Prog. Nucl. Magn. Reson. Spectrosc. 30, 101 (1997).

${ }^{4}$ D. Canet, S. Leclerc, M. Rocher, F. Guenneau, and D. Grandclaude, Appl. Magn. Reson. 22, 307 (2002).

${ }^{5}$ E. O. Stesjskal and J. E. Tanner, J. Chem. Phys. 42, 288 (1965).

${ }^{6}$ F. Humbert, M. Valtier, A. Retournard, and D. Canet, Appl. Magn. Reson. 134, 245 (1998).

${ }^{7}$ J. E. Tanner, J. Chem. Phys. 52, 2523 (1970).

${ }^{8}$ S. Leclerc, S. Bouguet-Bonnet, P. Mutzenhardt, J. Brondeau, and D. Canet, Magn. Reson. Chem. 41, 769 (2003). 